\title{
Short-Term Visual Recognition and Temporal Order Memory Are Both Well-Preserved in Aging
}

\author{
Robert Sekuler and Chris McLaughlin, \\ Brandeis University
}

\author{
Michael J. Kahana \\ University of Pennsylvania
}

\author{
Arthur Wingfield and Yuko Yotsumoto \\ Brandeis University
}

\begin{abstract}
Increased difficulty with memory for recent events is a well-documented consequence of normal aging, but not all aspects of memory are equally affected. To compare the impact of aging on short-term recognition and temporal order memory, young and older adults were asked to identify the serial position that a probe item had occupied in a study set, or to judge that the probe was novel (had not been in the study set). Stimuli were compound sinusoidal gratings, which resist verbal description and rehearsal. With retention intervals of 1 or 4 seconds, young and older adults produced highly similar overall performance, serial position curves, and proportions of trials on which a correct recognition response was accompanied by an incorrect temporal order judgment. Temporal order errors, which occurred on about one quarter of trials, were traced to two factors: perceptual similarity between the wrongly identified study item and the correct item, and temporal similarity between the wrongly identified item and the correct one. Our results show that short-term visual temporal order memory is well-preserved in normal aging, and when temporal order errors do occur, they arise from similar causes for young and older people.
\end{abstract}

Keywords: aging, short-term visual memory, temporal order memory, recognition, identification

Increased difficulty with memory for recent events is among normal aging's best known and most harmful consequences (Kausler, 1994; Wingfield \& Kahana, 2002). However, it is clear that not all dimensions of short-term memory are equally vulnerable to age-related changes (e.g., McIntosh et al., 1999; Olson et al., 2004). Source memory (Johnson, Hashtroudi, \& Lindsay, 1993), which includes information about the temporal order in which items were recently encountered, seems to be particularly vulnerable to the effects of aging (Newman, Allen, \& Kaszniak, 2001; Fabiani \& Friedman, 1997; Kahana, Howard, Zaromb, \& Wingfield, 2002, but see Siedlecki, Salthouse, \& Berish, 2005). To explore this topic further, we examined both visual short-term recognition memory and visual short-term temporal order memory, using rehearsal-resistant (Hwang et al., 2005) sinusoidal gratings as study and test items, and taking care to equate each participant's stimuli on the basis of visual discriminability.

Recent studies with such stimuli revealed a striking preservation of short-term, item recognition memory with aging (DellaMaggiore et al., 2000; Bennett, Sekuler, McIntosh, \& Della-

Robert Sekuler, Chris McLaughlin, Arthur Wingfield, and Yuko Yotsumoto, Volen National Center for Complex Systems, Brandeis University; Michael J. Kahana, Department of Psychology, University of Pennsylvania.

Supported by NIH Grants MH068404, MH55687, and AG15852, and by the W. M. Keck Foundation. We thank Feng Zhou for the supplementary experiment on visual confusions between stimuli.

Correspondence concerning this article should be addressed to Robert Sekuler, Volen National Center for Complex Systems, Brandeis University, Mailstop 013, Waltham, MA 02454. E-mail: sekuler@brandeis.edu
Maggiore, 2001; McIntosh et al., 1999). For example, Sekuler, Kahana, McLaughlin, Golomb, and Wingfield (2005) showed that young and older participants demonstrated equivalent short-term visual recognition memory, achieving equivalent proportions of correct old-new judgments, at each of three different, brief test delays. The present study adapted these basic methods to examine short-term temporal order memory with new samples of participants. In addition, we exploited the metric properties of the grating stimuli in order to identify deterministic causes of observed errors in short-term temporal order memory.

On each trial in our study, participants saw a series of three compound gratings followed by a probe (test) grating. These stimuli were used, in part, because of their resistance to consistent verbal labeling and rehearsal (Della-Maggiore et al., 2000; Hwang et al., 2005), which makes it possible to examine short-term visual memory with minimal contamination from verbal mediation. At the end of each trial, participants judged whether a probe item had been in the study series, and, if so, which study item it had been, first, second, or third. Because judgments of item familiarity (old or new) and judgments related to temporal order can be differentially affected by even short delays in testing (Yonelinas \& Levy, 2002), we made measurements with two different retention intervals. Our questions were: (a) is short-term visual memory for item familiarity better than short-term visual memory for temporal order information? and (b) might these aspects of visual short-term memory be preserved in aging, in contrast to other aspects of memory, including verbal memory (e.g., Kahana et al., 2002)? 


\section{Method}

\section{Procedure}

On each trial, a study series comprising three compound sinusoidal gratings was followed by a probe stimulus $(p)$. Each of the three study stimuli $\left(s_{1}, s_{2}\right.$, and $\left.s_{3}\right)$ was presented for $750 \mathrm{~ms}$, with interstimulus intervals of $400 \mathrm{~ms}$. Then, after a delay of either $1 \mathrm{~s}$ or $4 \mathrm{~s}$, a warning tone sounded, and $p$ was presented for $750 \mathrm{~ms}$. Participants used a response selection display to indicate whether $p$ had been in the study set and, if it had been in the set, which of the three study items it matched.

We refer to a $p$ that matched one of the study items as a target, and a trial on which $p$ matched a study item as a target trial. We designate a nonmatching $p$ as a lure, and we designate trials on which $p$ matched none of the study items, a lure trial. Target and lure trials occurred in random order, in a ratio of $3: 1$. For lure trials, stimuli were selected by randomly sampling four items (three study and a $p$ ) without replacement from the entire pool of 25 stimuli. For target trials, the three study items were randomly selected from the pool of 25 stimuli, but the choice of $p$ was constrained so that it matched one of the three study items in the study series, and did so equally often for items in the first, second, or third serial positions.

On each trial, after $p$ disappeared, a response selection screen was presented on the computer display and remained visible until the participant made a response. The selection screen displayed four alternatives, labeled none, first, second, or third. Participants used the computer mouse to select the alternative that corresponded to the serial position (first, second, or third) of the study stimulus, $s_{1}, s_{2}$, or $s_{3}$ that matched $p$. If $p$ seemed to have matched none of the study items, the participant clicked on the alternative labeled none. No instructions were given about response speed.

Distinctive tones provided feedback after each response. On target trials, feedback was contingent upon the response's identification component. Feedback signaled whether the participant's response correctly identified which study item $\left(s_{1}, s_{2}\right.$, or $\left.s_{3}\right)$ matched $p$; and, like incorrect identification responses, a none response on a target trial brought feedback that the response was wrong. On lure trials, feedback was contingent on whether the response correctly signified that none of the study items matched $p$; all other responses (i.e., first, second, or third) were followed by feedback that the response had been wrong.

Prior to the experiment, participants were told the proportions of target and lure trials. Each participant was tested on 288 trials distributed across two 1-hr sessions. In each session, participants completed a block of 72 trials with a preprobe delay of $1 \mathrm{~s}$, and another block of 72 trials with a preprobe delay of $4 \mathrm{~s}$. These were designated Conditions A and B, and half the participants completed four blocks of experimental trials in an ABBA order, half in a $\mathrm{BAAB}$ order.

\section{Participants}

Ten young adults $(19-25$ years of age, $M=21.8, S D=2.1)$ and 10 older adults (65-80 years of age, $M=71.4, S D=6.0)$ participated in this study for monetary compensation. Five of the young adults were male, five female; three of the older adults were male, seven female. Each participant's visual acuity was measured using Landolt $C$ targets. Young participants' acuity ranged from $20 / 13-20 / 25(M=20 / 18.1, S D=3.2)$ and older participants' acuity ranged from $20 / 22-20 / 40(M=20 / 31.7, S D=7.2)$. Contrast sensitivity was measured with a Lighthouse Letter Contrast Sensitivity Test. Young participants' sensitivity ranged from $1.64-1.80(M=$ $1.71, S D=0.07$ ) and older participants' sensitivity ranged from $1.28-1.68$ $(M=1.56, S D=0.12)$.

To control effects of individual or age-related differences in vision, a participant's stimuli were scaled according to that participant's discrimination threshold for spatial frequency (Zhou, Kahana, \& Sekuler, 2004). Thus, prior to our memory experiment, a staircase procedure measured each participant's spatial frequency discrimination threshold for sinusoidal gratings. Threshold was defined as the frequency difference corresponding to $p=.794$ on the psychometric function. The timing of stimulus presentation corresponded to the timing that would be used in the memory experiment itself. The resulting Weber fractions ranged from $6.5 \%-13.2 \%$ for young participants $(M=9.7, S D=3.5)$, and from $8.2 \%-26.0 \%$ for older participants $(M=16.7, S D=6.6)$. The difference between group means was significant, $t(18)=-2.98, p<.01$.

By self-report, all older participants were in good health, had good cognitive function, and had at least some college education. Older participants' score on the Mini-Mental State Examination ranged from 28-30, with a $M=29.4, S D=.07$, which exceeds the population-based norm of 28 for 65-79 year olds with college experience or an advanced degree (Crum, Anthony, Bassett, \& Folstein, 1993).

\section{Stimuli}

Stimuli for each trial were drawn from a pool of compound sinusoidal gratings, each comprising superimposed vertical and horizontal sinusoidal luminance gratings. Details are given in Sekuler et al. (2005). Crossing five vertical spatial frequencies with five horizontal spatial frequencies generated the pool of stimuli for each participant. Vertical as well as horizontal spatial frequencies were 2 cycles/degree plus or minus three times or six times a participant's Weber fraction for spatial frequency.

The gratings' sinusoidal components had a Michelson contrast of 0.2 , a value well above the threshold for detection. To minimize edges, stimuli were windowed by a circular 2-D Gaussian with space constant of 1 degree visual angle. The display's mean luminance was fixed at $17.8 \mathrm{~cd} / \mathrm{m}^{2}$. Each participant viewed the stimulus from a distance of $114 \mathrm{~cm}$, with head supported and steadied by a head rest and chin cup. Trials were self paced.

By enforcing a minimum between-stimulus difference of three times a participant's discrimination threshold, we reduced the likelihood that perceptual confusions between pairs of stimuli, with minimal memory load, could by themselves lead to misidentifications. With threshold defined by the point $p=.794$ on the psychometric function, stimuli that differed by one threshold unit would be mistaken for one another with $p=1-0.794$, or 0.206 . By extrapolation, stimuli that differed by three times the threshold would be mistaken for one another $0.206^{3}$, or slightly less than $1 \%$ of trials. To check this prediction, a supplementary experiment tested five new participants, ages 18-27. With pairs of gratings whose spatial frequencies differed by three times a participant's discrimination threshold, perceptual confusions did indeed occur on less than $1 \%$ of trials.

\section{Results}

Table 1 summarizes the principal measures used to characterize participants' mnemonic processes. The table's first row shows the proportion of correct identifications of serial position; that is, the proportion of trials on which the stimulus was $s_{\mathrm{t}}$, where $s_{\mathrm{t}} \in\{1,2$, $3\}$, and participants responded $r_{\mathrm{t}}$, where $r_{\mathrm{t}} \in\{1,2,3\}$. Table 1's second row gives the proportion of correct recognitions calculated without regard to identification of serial position. This is the

Table 1

Mean Proportions and Standard Errors of Measurement for Basic Response Measures

\begin{tabular}{lcc}
\hline \multicolumn{1}{c}{ Measure } & Young participants & Older participants \\
\hline $\mathrm{P}$ (correct identification) & $0.61 \pm .03$ & $0.65 \pm .02$ \\
$\mathrm{P}$ (correct recognition) & $0.85 \pm .02$ & $0.90 \pm .01$ \\
$\mathrm{P}$ (identification $\mid$ recognition) & $0.79 \pm .03$ & $0.83 \pm .03$ \\
$\mathrm{P}\left(r_{1} \cap r_{2} \cap r_{3}\right)$ & $0.77 \pm .02$ & $0.81 \pm .02$ \\
\hline
\end{tabular}


proportion of target trials on which participants responded either $r_{1}, r_{2}$, or $r_{3}$ given $s_{\mathrm{t}}$, aggregating cases in which the response was $r_{\mathrm{t}}$ and cases in which it was not. The third row in the table gives a conditional value, $r_{t} \mid s_{t}$, the proportion of trials on which both the recognition judgment (yes) and the identification of $p$ 's serial position were correct. If every correct recognition had been accompanied by a correct identification, cells in the third row would show $\mathrm{P}$ (Identification|Recognition) $=1$. Target trials on which recognition was correct, but identification wrong constitute mis- identifications, that is, errors in serial position judgment. The proportion of such trials can be obtained from the quantity 1-P(Identification|Recognition). The last row in Table 1 gives the proportion of all trials, right or wrong, on which participants judged that $p$ had been in the study series. The difference between groups on this measure was not significantly different. For comparison, the actual proportion of target trials was 0.75 .

Figure $1 \mathrm{~A}$ and $1 \mathrm{~B}$ show the proportion of correct recognitions and correct identifications of serial position for all three serial

\section{Young Participants}
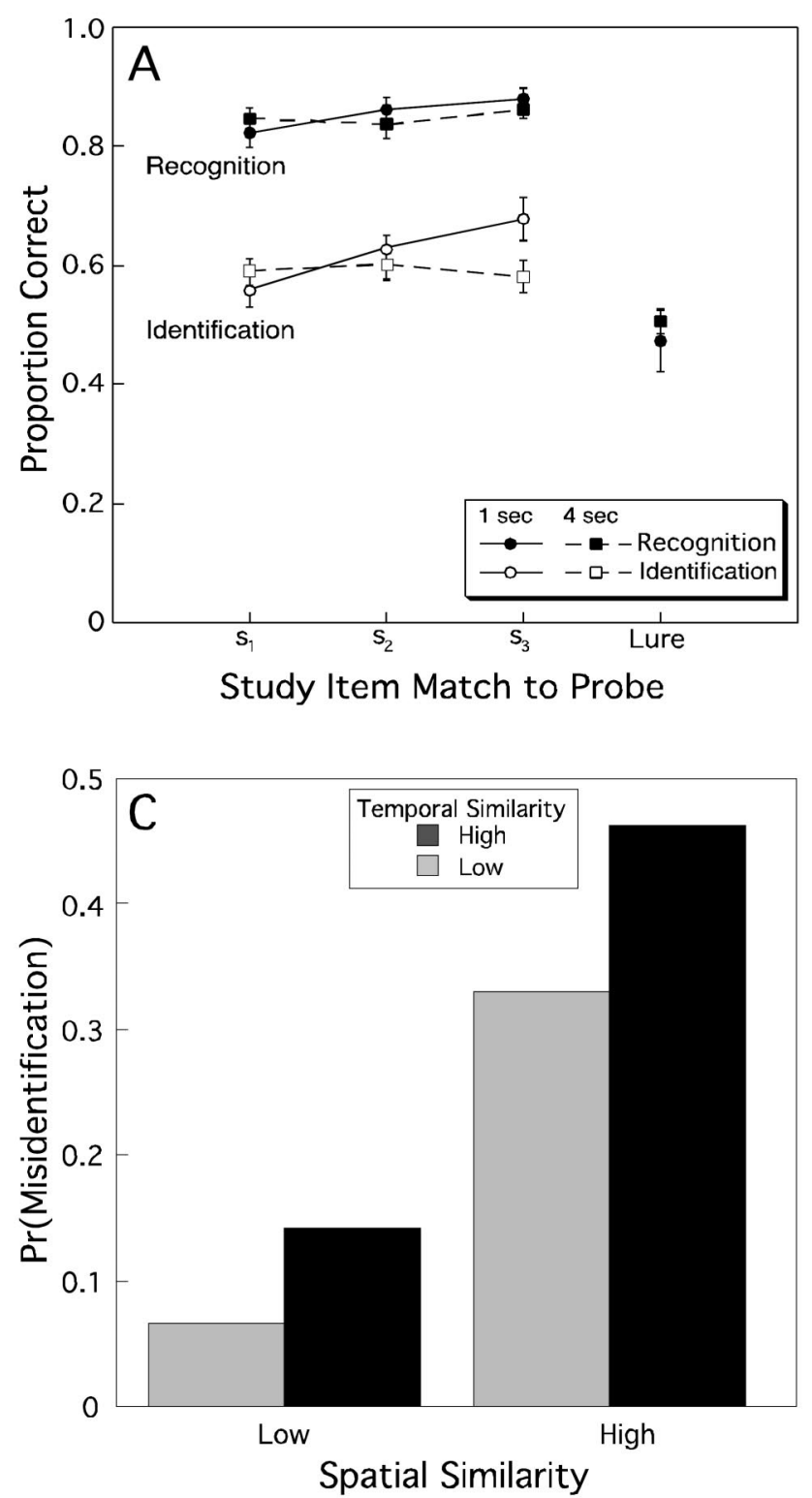

\section{Older Participants}
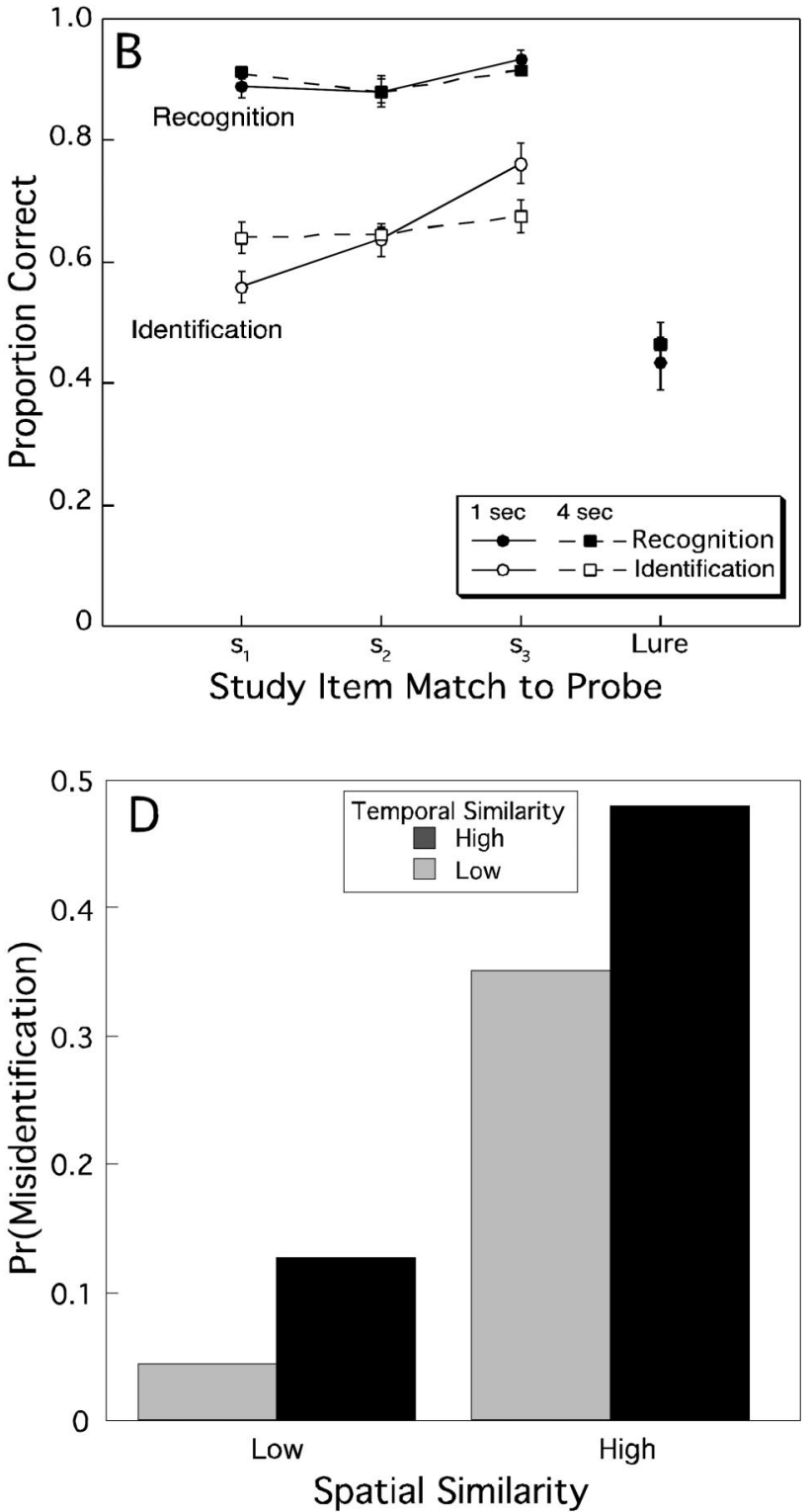

Figure 1. Proportions of correct recognition and identification for young (A) and older (B) participants. Also shown are proportions of items misidentified on target trials by young participants $(\mathrm{C})$ and by older participants (D) as a function of the spatial and temporal similarity between correct and misidentified study stimuli. In 1C and 1D, two levels of spatial difference are plotted on the $x$-axis. Each is plotted separately for stimulus pairs that were temporally similar (black bars), and for pairs that were temporally dissimilar (gray bars). 
positions and for lure trials (trials on which $p$ matched none of the study items). Results for young participants are shown in Figure 1A; results for older participants appear in Figure 1B. In addition, results are separated according to retention interval, $1 \mathrm{~s}$ versus $4 \mathrm{~s}$. There was no systematic difference in visual short-term recognition between the two retention intervals, 1 and $4 \mathrm{~s}$. In general, older participants achieved a somewhat higher proportion of correct recognitions than did their younger counterparts. Previous memory studies have shown that older participants' performance can be distorted by a criterion change (Harkins, Chapman, \& Eisdorfer, 1979; Kosslyn, Thompson, Kim, \& Alpert, 1995; Trahan, Larrabee, \& Levin, 1986). Therefore, we evaluated potential criterion changes, taking account not only of correct recognition responses, but also the correct rejection rate. When this was done, we found that the modest age-dependent superiority in recognition was accompanied by a corresponding decrease in the rate of correct rejection rate for a lure, that is for a $p$ that matched none of the study items. Finally, when pairs of hits and false alarms were expressed as $d^{\prime}$ values (Macmillan \& Creelman, 2005), the mean $d^{\prime}$ s for the young and older participants were 1.01 and 1.16, respectively. Bootstrap resampling showed that in the absence of any actual difference between the two groups, a difference this large or larger would occur with $p>.30$. The nonsignificance between the groups' $d$ 's strengthens the claim that the two age groups had essentially equivalent recognition performance. Because neither the main effect of age nor any interaction involving age were statistically reliable, the following discussion ignores that variable.

Confirming what may be evident in the first and second rows of Table 1, Figure 1 shows that the proportion of correct recognitions was appreciably higher than the proportion of correct identifications of serial position, $F(1,18)=395.13, p<.01$. This difference reflects the influence of errors in identifying the serial position of recognized items.

Although recognition responses showed no statistically significant serial position effect, such an effect was manifest with identification responses, that is, when participants' serial position judgments were taken into account. This serial position effect was considerably diminished at the longer of our two retention intervals, $F(2,36)=6.22, p<.01$.

Errors in visual temporal order judgments can be exploited to identify the information that participants use to make successful judgments. This is especially true when, as is the case here, the metric properties of stimuli make it possible to relate misidentifications to stimulus characteristics. For young participants, on $21 \%$ of trials when recognition was correct, the target was attributed to an incorrect serial position; for older participants, the corresponding value was $17 \%$. The difference between the two age groups was not statistically significant $(p>.40)$.

Basically, misidentifications could have arisen from either of two quite distinct sources. Some or all of the misidentifications could have been entirely stochastic, reflecting random guesses made when participants had no actual usable memory of what had been seen. Alternatively, misidentifications could have come from some deterministic process, for example, systematic errors associated with partial loss of serial position information. We set out to evaluate these alternative accounts of misidentifications.

To compare competing stochastic and deterministic accounts of misidentifications, each participant's misidentifications were sorted into the cells of a notional $2 \times 2$ table. In this sorting, we considered only trials on which $p$ matched $s_{1}$ or $s_{3}$. The table's rows corresponded to two levels of a variable we call spatial similarity; the table's columns correspond to two levels of a variable we call temporal similarity. To generate the value of spatial similarity, we compared the Euclidean distance in spatial frequency between (a) $p$ and the misidentified study item, and (b) $p$ and the remaining study item that did not match $p$. If the first of these two distances was the smaller, we categorized spatial similarity between $p$ and the misidentified study item as high; otherwise, we categorized spatial similarity as low. For temporal similarity, we categorized misidentifications according to whether the error in identification represented a shift of one (high similarity) or two (low similarity) serial positions. For example, if $s_{2}$ was misidentified as matching $p$, when the actual matching study item was $s_{3}$, this error of one serial position was categorized as high temporal similarity; if $s_{1}$ was misidentified as matching $p$, when the actual matching study item was $s_{3}$, the error of two serial positions was categorized as low temporal similarity. A factorial cross of spatial and temporal variables produced four combinations of spatiotemporal differences between $p$ and the misidentified study item.

The proportions of all serial position errors that fell into each of the four categories are shown in Figure 1C for young participants, and in Figure 1D for the older participants. On the horizontal-axis in each panel is the category, low or high, of spatial similarity; black bars show results with high temporal similarity, and gray bars show results with low temporal similarity. Because the distribution of misidentifications across the four categories was essentially the same for both age groups, for now we ignore the age variable.

The results shown in Figures 1C and 1D allow us to dismiss the hypothesis that all misidentifications resulted from a stochastic process. A process in which participants guessed randomly would on average have produced four bars of roughly equal height in Figure 1C and 1D. Monte Carlo simulations show that a stochastic process would generate results as biased as those we observed in fewer than 1 in 100,000 replications of the experiment. Although we cannot rule out the possibility that random guesses produced some of the misidentifications, we can assert that many misidentifications arose from deterministic processes.

The distribution of misidentifications across the four categories in the notional $2 \times 2$ table suggests that both temporal and physical similarity induced temporal order errors, with physical similarity exerting a larger effect than temporal similarity (compare the pair of bars at the left side of Figure 1C or Figure 1D to the corresponding ones at the figure's right). The figure shows no evidence of an interaction between the two variables: the black and gray bars at the left side of Figure $1 \mathrm{C}$ differ by about as much as the corresponding bars at the Figure 1C's right side. So, the same processes appear to be at work with both young and older participants, and they have quite comparable effects on both groups.

Finally, we must comment on one deterministic process that had the potential to promote errors in short-term temporal order memory, but most likely did not: purely perceptual confusions among stimuli. Stimulus series were constructed so that any two study stimuli differed in spatial frequency by at least three times a participant's difference threshold. As noted earlier, when visual stimuli differ by that much, perceptual confusion alone, with 
minimal contribution from errors in short-term memory, would have caused the stimuli to be mistaken for one another less than $1 \%$ of the time. So perception-based mistaken identity alone cannot explain the much higher proportion of misidentifications, that is, the obtained values of 1-P(Identification|Recognition). Instead, we must look elsewhere for an explanation of misidentifications.

\section{Discussion}

Sensory researchers have long understood that perceptual errors, including illusions, can be a valuable source of insight into perception's normal operation (Eagleman, 2001). In the same way, from the very beginning of systematic research on memory, errors and failures have been useful in illuminating memory's normal operation (Schacter \& Dodson, 2001). As Figure 1 suggested, both spatial and temporal similarity promote misidentifications of serial position, and that these separate effects are approximately additive. How, though, might these influences operate?

To understand how spatial similarity might lead to errors in temporal order, we will use the basic structure of summedsimilarity (or global-matching) memory models (Nosofsky, 1986; Clark \& Gronlund, 1996; Kahana \& Sekuler, 2002). We will work within this framework, because one member of this class of models, Noisy Exemplar Model (NEMo), has already accounted successfully for short-term memory with stimuli like those used here (Kahana \& Sekuler, 2002; Kahana, Zhou, Geller, \& Sekuler, in press). A summed similarity model assumes that the study items, $s_{1} \ldots s_{3}$, are stored in memory as corresponding noisy exemplars, $m_{1} \ldots m_{3}$, where the exemplars' subscripts signify the order in which the visual stimuli had been presented. When the probe, $p$, is presented, $\eta_{1} \ldots \eta_{3}$, the set of similarities between $p$ and each of the noisy exemplars is computed. Again, subscripts signify the order in which study stimuli were presented. NEMo describes each similarity value as an exponentially decreasing function of the spatial frequency difference between $p$ and the corresponding values, $m_{1} \ldots m_{3}$. From the resulting similarity values, a summed similarity, $\Sigma \eta$, is computed. Some criterion value, $\Sigma_{\eta}>k$, is taken as evidence that at least one of the study items matched $p$, which has made $p$ seem familiar. Over trials, the probability of a recognition response (e.g., a yes response) corresponds to the proportion of trials on which $\Sigma_{\eta}>k$.

The central role that noise can play in summed similarity models brings to mind the role it occupies in some theories of cognitive aging. For example, Welford (1984) offered the influential proposal that various age-related changes in performance reflected older adults' relatively higher levels of internal neural noise (random variability). Researchers have developed and deployed powerful computational methods for testing detailed descriptions of noise's role in short-term memory, distinguishing between different sources of noise, such as internal versus external noise, and different forms of noise, such as multiplicative versus additive noise (Gold, Murray, Sekuler, Bennett, \& Sekuler, 2005), but such analyses have as yet not been applied to results from older adults. Because we intentionally tailored stimuli to individual participants' visual discrimination, it is impossible to tell from our data whether age-related differences in internal noise accompany visual encoding. But, we can exploit the summed similarity framework to generate useful propositions about age-related noise in the context of short-term recognition and temporal order memory.
First, consider how a summed similarity model could be extended to account for temporal order judgments. This extension requires that the model perform one additional operation on the set of similarities between a trial's study stimuli and $p$. In this additional operation, the similarities are processed with a max operator, which returns two values, the largest item $\in\left\{\eta_{1} \ldots \eta_{3}\right\}$, and the index (serial position) of that largest item. Hereafter, we refer to these returned quantities as value and index, respectively. The identification of the matching serial position is determined by the index, $1 \ldots 3$, returned by the max operator. Because of the noise associated with each exemplar, there will be trials on which the index returned by $\max$ would not represent the serial position whose study item physically matched $p$, but would represent instead the serial position of another study item. On such trials, the model generates a temporal order error, in which the serial position of the matching item is misidentified. The probability of such errors will be some monotonically decreasing function of each study item's similarity in spatial frequency to $p$. In other words, study items that did not match $p$, but were spatially similar to it would be more likely misidentified as a match than would study items that were less spatially similar to $p$. Of course, this is the pattern of spatial similarity effects shown in Figure 1C and 1D, and suggests that once stimuli have been adjusted for small age-related differences in discriminability, there are little or no age-related differences in the internal noise in short-term memory that produces spatial-similarity based, temporal order lapses.

Second, consider how a different mechanism is required to motivate temporal similarity's influence on misidentifications of serial position. Drawing upon accounts of temporal effects in free recall (Howard \& Kahana, 1999, 2002), we assume that the representation of each noisy exemplar is tagged in memory with a temporal code, and that each item's temporal tag could be misassigned at encoding, or degraded in memory by passage of time and/or interference. If such degradation were partial rather than complete (Dodson, Holland, \& Shimamura, 1998), serially adjacent positions in a sequence would more likely be confused with one another than would positions more widely separated in a sequence. In the case at hand, with partial loss of serial position information, $s_{1}$ is more likely misremembered as $s_{2}$ than as $s_{3}$, and $s_{3}$ is more likely misremembered as $s_{2}$ than as $s_{1}$. Again, this is the pattern of results seen in Figure 1C and 1D. We should note that this effect resembles Dodson and colleagues' (1998) demonstration that even when individuals misidentify the source of information, they may retain partial information about that source. Clearly, our results suggest that young and older adults retain this information in equal measure.

With the rapid presentations and relatively short retention intervals used in this experiment, it is not likely that by itself the passage of time was primarily responsible for degrading serial position information (e.g., Sekuler et al., 2005). Instead, interference in memory, generated by presentation of successive study items was more likely at fault. Whatever the details of such interference prove to be, the present results demonstrate a striking age invariance in both item and order short-term memory for visual gratings, which resist verbal labeling and rehearsal. This invariance stands in contrast to age-related differences in order memory with verbal material (Kahana et al., 2002). This divergence leads us to hypothesize that our results depend crucially upon test materials whose discriminability is equated between 
participants (Zhou et al., 2004), and whose characteristics minimize participants' reliance on verbal labeling and rehearsal. Further speculation about our results' boundary conditions must await future research. However, it does seem that in spite of welldocumented, age-related declines in other cognitive domains, the neural substrates underlying short-term visual memory remain robust, perhaps supported by compensatory reorganization of taskrelated cortical networks (McIntosh et al., 1999; Della-Maggiore et al., 2000).

\section{References}

Bennett, P. J., Sekuler, A. B., McIntosh, A. R., \& Della-Maggiore, V. (2001). The effects of aging on visual memory: Evidence for functional reorganization of cortical networks. Acta Psychologia (Amsterdam), 107(1-3), 249-273.

Clark, S. E., \& Gronlund, S. D. (1996). Global matching models of recognition memory: How the models match the data. Psychonomic Bulletin \& Review, 3, 37-60.

Crum, R. M., Anthony, J. C., Bassett, S. S., \& Folstein, M. F. (1993). Population-based norms for the Mini-Mental State Examination by age and education level. Journal of the American Medical Association, 269, 2386-2391.

Della-Maggiore, V., Sekuler, A. B., Grady, C. L., Bennett, P. J., Sekuler, R., \& McIntosh, A. R. (2000). Corticolimbic interactions associated with performance on a short-term memory task are modified by age. Journal of Neuroscience, 20(22), 8410-8416.

Dodson, C., Holland, P., \& Shimamura, A. (1998). On the recollection of specific- and partial-source information. Journal of Experimental Psychology: Learning, Memory, and Cognition, 24, 1121-1136.

Eagleman, D. (2001). Visual illusions and neurobiology. Nature Reviews Neuroscience, 2(12), 920-926.

Fabiani, M., \& Friedman, D. (1997). Dissociations between memory for temporal order and recognition memory in aging. Neuropsychologia, $35(2), 129-141$.

Gold, J. M., Murray, R. F., Sekuler, A. B., Bennett, P. J., \& Sekuler, R. (2005). Visual memory decay is deterministic. Psychological Science, 16, 769-774.

Harkins, S. W., Chapman, C. R., \& Eisdorfer, C. (1979). Memory loss and response bias in senescence. Journal of Gerontology, 34, 66-72.

Howard, M., \& Kahana, M. J. (1999). Contextual variability and serial position effects in free recall. Journal of Experimental Psychology: Learning, Memory, and Cognition, 25, 923-941.

Howard, M. W., \& Kahana, M. J. (2002). When does semantic similarity help episodic retrieval? Journal of Memory \& Language, 46(1), 85-98.

Hwang, G. M., Jacobs, J., Geller, A., Danker, J., Sekuler, R., \& Kahana, M. J. (2005). EEG correlates of verbal and nonverbal working memory. Behavioral and Brain Function, 1, 20-26.

Johnson, M. K., Hashtroudi, S., \& Lindsay, D. S. (1993). Source monitoring. Psychological Bulletin, 114(1), 3-28.

Kahana, M. J., Howard, M. W., Zaromb, F., \& Wingfield, A. (2002). Age dissociates recency and lag recency effects in free recall. Journal of Experimental Psychology: Learning, Memory, and Cognition, 28, 530540.
Kahana, M. J., \& Sekuler, R. (2002). Recognizing spatial patterns: A noisy exemplar approach. Vision Research, 42, 2177-2192.

Kahana, M. J., Zhou, F., Geller, A. \& Sekuler, R. (in press). Lure-similarity affects visual episodic recognition: Detailed tests of a noisy exemplar model. Memory \& Cognition.

Kausler, D. M. (1994). Learning and memory in normal aging. San Diego: Academic Press.

Kosslyn, S., Thompson, W. L., Kim, I. J., \& Alpert, N. M. (1995). Topographical representations of mental images in primary visual cortex. Nature, 378(6556), 496-498.

Macmillan, N. A., \& Creelman, C. D. (2005). Detection theory: A user's guide (2nd ed.). Mahwah, NJ: Erlbaum.

McIntosh, A. R., Sekuler, A. B., Penpeci, C., Rajah, M. N., Grady, C. L., Sekuler, R., \& Bennett, P. (1999). Recruitment of unique neural systems to support visual memory in normal aging. Current Biology, 9(21), $1275-1278$.

Newman, M. C., Allen, J. J. B., \& Kaszniak, A. W. (2001). Tasks for assessing memory for temporal order versus memory for items in aging. Aging, Neuropsychology \& Cognition, 8(1), 72-78.

Nosofsky, R. M. (1986). Attention, similarity, and the identification categorization relationship. Journal of Experimental Psychology: General, $115,39-57$.

Olson, I. R., Zhang, J. X., Mitchell, K. J., Johnson, M. K., Bloise, S. M., \& Higgins, J. A. (2004). Preserved spatial memory over brief intervals in older adults. Psychology and Aging, 19, 310-317.

Schacter, D., \& Dodson, C. (2001). Misattribution, false recognition, and the sins of memory. Philosophical Transactions of the Royal Society of London, Series B, 356, 1385-1393.

Sekuler, R., Kahana, M. J., McLaughlin, C., Golomb, J., \& Wingfield, A. (2005). Preservation of episodic visual memory in aging. Experimental Aging Research, 31, 1-12.

Siedlecki, K. L., Salthouse, T. A., \& Berish, D. E. (2005). Is there anything special about the aging of source memory? Psychology and Aging, 20, $19-32$.

Trahan, D. E., Larrabee, G. J., \& Levin, H. S. (1986). Age-related differences in recognition memory for pictures. Experimental Aging Research, $12,147-150$.

Welford, A. T. (1984). Between bodily changes and performance: Some possible reasons for slowing with age. Experimental Aging Research, 102, 73-88.

Wingfield, A., \& Kahana, M. J. (2002). The dynamics of memory retrieval in older adulthood. Canadian Journal of Psychology, 56, 187-199.

Yonelinas, A., \& Levy, B. (2002). Dissociating familiarity from recollection in human recognition memory: Different rates of forgetting over short retention intervals. Psychonomic Bulletin \& Review, 9(3), 575582.

Zhou, F., Kahana, M. J., \& Sekuler, R. (2004). Short-term episodic memory for visual textures: A roving probe gathers some memory. Psychological Science, 15(26), 112-118.

Received December 1, 2004 Revision received January 3, 2006 Accepted January 23, 2006 